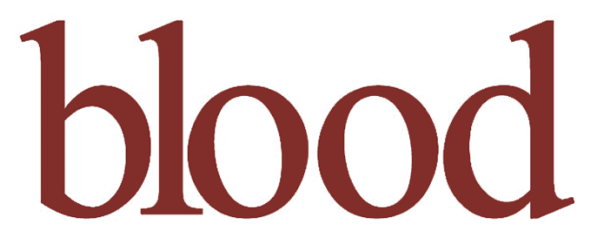

2007 109: 1834-1840

Prepublished online October 31, 2006:

doi:10.1182/blood-2006-06-032276

\title{
Bone morphogenic protein antagonist Drm/gremlin is a novel proangiogenic factor
}

Helena Stabile, Stefania Mitola, Emanuela Moroni, Mirella Belleri, Stefania Nicoli, Daniela Coltrini, Francesco Peri, Antonello Pessi, Laura Orsatti, Fabio Talamo, Vincent Castronovo, David Waltregny, Franco Cotelli, Domenico Ribatti and Marco Presta

Updated information and services can be found at:

http://bloodjournal.hematologylibrary.org/content/109/5/1834.full.html

Articles on similar topics can be found in the following Blood collections

Chemokines, Cytokines, and Interleukins (564 articles)

Free Research Articles (1399 articles)

Hemostasis, Thrombosis, and Vascular Biology (2497 articles)

Information about reproducing this article in parts or in its entirety may be found online at: http://bloodjournal.hematologylibrary.org/site/misc/rights.xhtml\#repub_requests

Information about ordering reprints may be found online at:

http://bloodjournal.hematologylibrary.org/site/misc/rights.xhtml\#reprints

Information about subscriptions and ASH membership may be found online at:

http://bloodjournal.hematologylibrary.org/site/subscriptions/index.xhtml

Blood (print ISSN 0006-4971, online ISSN 1528-0020), is published weekly by the American Society of Hematology, 2021 L St, NW, Suite 900,

Washington DC 20036.

Copyright 2011 by The American Society of Hematology; all rights reserved.

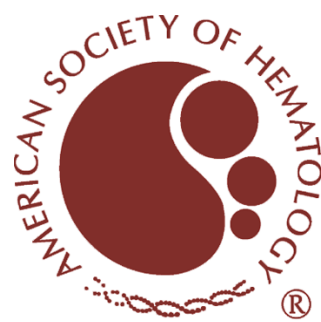




\title{
Bone morphogenic protein antagonist Drm/gremlin is a novel proangiogenic factor
}

\author{
Helena Stabile, ${ }^{1}$ Stefania Mitola, ${ }^{1}$ Emanuela Moroni, ${ }^{1}$ Mirella Belleri, ${ }^{1}$ Stefania Nicoli, ${ }^{1}$ Daniela Coltrini, ${ }^{2}$ \\ Francesco Peri, ${ }^{3}$ Antonello Pessi, ${ }^{4}$ Laura Orsatti, ${ }^{4}$ Fabio Talamo, ${ }^{4}$ Vincent Castronovo, ${ }^{5}$ David Waltregny, ${ }^{6}$ \\ Franco Cotelli, ${ }^{7}$ Domenico Ribatti, ${ }^{8}$ and Marco Presta ${ }^{1}$
}

${ }^{1}$ Unit of General Pathology and Immunology, Department of Biomedical Sciences and Biotechnology, University of Brescia, Italy; ${ }^{2}$ Unit of Histology, Department of Biomedical Sciences and Biotechnology, University of Brescia, Italy; 3Department of Biotechnology and Biosciences, University of Milan-Bicocca, Milan, Italy; ${ }^{4}$ Istituto di Ricerche di Biologia Molecolare P. Angeletti, Pomezia, Rome, Italy; ${ }^{5}$ Metastasis Research Laboratory, Center of Experimental Cancer Research, University of Liège, Belgium; ${ }^{6}$ Department of Pathology, Center of Experimental Cancer Research, University of Liège, Belgium; ${ }^{7}$ Department of Biology, University of Milan, Italy; ${ }^{8}$ Department of Human Anatomy and Histology, University of Bari, Italy

\begin{abstract}
Angiogenesis plays a key role in various physiologic and pathologic conditions, including tumor growth. Drm/gremlin, a member the Dan family of bone morphogenic protein (BMP) antagonists, is commonly thought to affect different processes during growth, differentiation, and development by heterodimerizing various BMPs. Here, we identify Drm/gremlin as a novel proangiogenic factor expressed by endothelium. Indeed, Drm/gremlin was purified to homogeneity from the conditioned medium of transformed endothelial cells using an endothelial-cell sprout-
\end{abstract}

ing assay to follow protein isolation. Accordingly, recombinant Drm/gremlin stimulates endothelial-cell migration and invasion in fibrin and collagen gels, binds with high affinity to various endothelial cell types, and triggers tyrosine phosphorylation of intracellular signaling proteins. Also, Drm/gremlin induces neovascularization in the chick embryo chorioallantoic membrane. BMP4 does not affect Drm/gremlin interaction with endothelium, and both molecules exert a proangiogenic activity in vitro and in vivo when administered alone or in combination.
Finally, Drm/gremlin is produced by the stroma of human tumor xenografts in nude mice, and it is highly expressed in endothelial cells of human lung tumor vasculature when compared with nonneoplastic lung. Our observations point to a novel, previously unrecognized capacity of Drm/gremlin to interact directly with target endothelial cells and to modulate angiogenesis. (Blood. 2007;109: 1834-1840)

๑ 2007 by The American Society of Hematology

\section{Introduction}

Gremlin, also known as Drm (Down-regulated by v-mos), belongs to the Dan family of cysteine knot-secreted proteins., ${ }^{1,2} \mathrm{Drm} /$ gremlin exerts a potent bone morphogenic protein (BMP) antagonist activity by binding BMP2, BMP4, and BMP7 and preventing their interaction with cell-surface receptors. ${ }^{3}$ This capacity is thought to be responsible for the pattern-inducing activity of Drm/gremlin during embryonic development ${ }^{4}$ and to play a role in human diseases. ${ }^{5}$ However, intracellular, BMP-independent mechanism(s) of action ${ }^{6}$ may mediate the ability of Drm/gremlin to suppress transformation and tumorigenesis under certain experimental conditions. ${ }^{7}$ Also, Drm/gremlin interacts with Slit proteins and acts as a negative regulator of monocyte chemotaxis, thus suggesting a role for this protein in inflammation and immunity. ${ }^{8}$ Taken together these observations indicate that Drm/gremlin may exert multiple functions in different physiopathologic conditions via BMP-dependent and BMP-independent mechanisms of action. ${ }^{1-5}$ Nevertheless, the possibility that Drm/gremlin may exert a direct effect on target cells has never been explored.

Angiogenesis, the process of new blood vessel formation from preexisting ones, plays a key role in various physiologic and pathologic conditions, including inflammation and tumor growth. ${ }^{9}$ The local, continuous release of angiogenic growth factors and/or

Submitted June 28, 2006; accepted October 16, 2006. Prepublished online as Blood First Edition Paper, October 31, 2006; DOI 10.1182/blood-200606-032276.

The online version of this article contains a data supplement. alterations of the production of natural angiogenic inhibitors ${ }^{10}$ are responsible for the uncontrolled endothelial-cell proliferation that takes place during tumor neovascularization and in angiogenesisdependent diseases. ${ }^{11}$ Numerous inducers of angiogenesis have been identified, including members of the vascular endothelial growth factor (VEGF) ${ }^{12,13}$ and of the fibroblast growth factor (FGF) families. ${ }^{14}$ These angiogenic growth factors induce a complex "proangiogenic phenotype" in endothelial cells that recapitulates several aspects of the in vivo angiogenesis process (summarized in Javerzat et $\mathrm{al}^{15}$ ).

To elucidate the molecular determinants of endothelial-cell activation during angiogenesis, we originated a stable mouse aortic endothelial (MAE) cell line transfected with a human $F G F 2$ cDNA (FGF2-T-MAE cells). ${ }^{16,17}$ Transfectants are characterized by the overexpression of numerous genes implicated in the modulation of cell growth, differentiation, cell adhesion, and stress/ survival. ${ }^{18}$ FGF2-T-MAE cells are angiogenic and cause the formation of opportunistic vascular lesions by recruiting endothelial cells of the host. ${ }^{16,19}$ Accordingly, FGF2-T-MAE cells release an endothelial-cell motogen that appears to be distinct from other well-characterized angiogenic growth factors, including FGF2 and VEGF. ${ }^{19}$ 
Here, we describe the purification of this factor from the conditioned medium (CM) of FGF2-T-MAE cells and its identification as the Drm/gremlin protein. Our data demonstrate for the first time that Drm/gremlin plays a BMP-independent role in the angiogenic process by binding the endothelial cell surface, thus activating intracellular signaling and cell motility. Accordingly, Drm/gremlin induces new vessel growth in the chick embryo chorioallantoic membrane. Also, the expression of Drm/gremlin in the endothelium of human lung tumor specimens points to a role for this protein in blood vessel development in human cancers.

\section{Materials and methods}

\section{Cell cultures}

Immortalized Balb/c MAE cells were obtained from R. Auerbach (University of Wisconsin, Madison) and grown in Dulbecco modified minimal essential medium (DMEM; Gibco Life Technologies, Rockville, MD) combined with 10\% FCS (Gibco Life Technologies). FGF2-T-MAE cells were grown in DMEM supplemented with $4 \mathrm{mM}$ glutamine (Gibco Life Technologies) and 10\% FCS. Bovine aortic endothelial (BAE) cells and normal subcutaneous microvascular endothelial (SIE) cells ${ }^{20}$ (both provided by A. Vecchi, Istituto Mario Negri, Milan, Italy) were cultured in DMEM supplemented with $10 \%$ heat-inactivated donor calf serum. Human umbilical vein endothelial (HUVE) cells were cultured in EGM-2 medium (Clonetics, Palo Alto, CA).

\section{Drm/gremlin purification}

Conditioned medium (CM) was prepared by incubating confluent FGF2-TMAE cell cultures grown in 10-cm dishes with $8 \mathrm{~mL}$ serum-free DMEM for 2 to 3 days. CM (21 L) was precipitated with $70 \%$ of ammonium sulfate. Then, the protein precipitate was dissolved in $40 \mathrm{~mL}$ of $25 \mathrm{mM}$ MES/NaOH (pH 6.5) plus $1.0 \mathrm{mM}$ PMSF and dialyzed against the same buffer. The dialyzed protein fraction $(2 \mathrm{mg} / \mathrm{mL})$ was applied onto a $80-\mathrm{mL}$ SpSepharose Fast Flow column (Amersham Bioscience, Uppsala, Sweden) pre-equilibrated with $50 \mathrm{mM} \mathrm{MES} / \mathrm{NaOH}(\mathrm{pH}$ 6.5). The column was extensively washed with $50 \mathrm{mM} \mathrm{MES} / \mathrm{NaOH}(\mathrm{pH} 7.5)$ and eluted at a flow rate of $30 \mathrm{~mL} /$ hour with a linear 0 to $1.0 \mathrm{M} \mathrm{NaCl}$ gradient at $4^{\circ} \mathrm{C}$. The eluate was collected in 10-mL fractions, and aliquots from each fraction were assayed for their ability to stimulate MAE-cell sprouting in 3-dimensional fibrin gel. ${ }^{19}$ The biologically active fractions eluted at 0.8 to $0.9 \mathrm{M} \mathrm{NaCl}$ (see Figure 1B) were pooled together (10 mg total protein) and concentrated 10 times with a $30-\mathrm{kDa}$ cutoff ultrafiltration system (Amicon, Bedford, MA). Proteins were then loaded onto a 10-mL heparin-Sepharose column equilibrated in $10 \mathrm{mM}$ Mes/ $\mathrm{NaOH}(\mathrm{pH} 7.0)$ and eluted with a linear 0 to $1.0 \mathrm{M} \mathrm{NaCl}$ gradient at $4^{\circ} \mathrm{C}$ at a flow rate of $1.0 \mathrm{~mL} /$ minute. The major peak of activity eluted at $1.0 \mathrm{M} \mathrm{NaCl}$ (Figure 1C). Pooled fractions, containing 250 to $500 \mu \mathrm{g}$ total proteins, were concentrated with a $50-\mathrm{kDa}$ cutoff centrifugal concentrator (Centriplus; Millipore, Bedford, MA) and freezedried. Next, the sample was dissolved in $1.0 \%$ trifluoroacetic acid and loaded onto a $1.0-\mathrm{mL}$ C4 Symmetry 300 column (Waters, Milford, MA) equilibrated with $0.1 \%$ trifluoroacetic acid $(1.0 \mathrm{~mL} /$ minute) for the initial 30 minutes. Bound material was eluted at $1.0 \mathrm{~mL} /$ minute using a 40 -minute linear $0 \%$ to $50 \%$ acetonitrile gradient in $0.1 \%$ trifluoroacetic acid (Figure 1D). Collected fractions were freeze-dried, resuspended in $25 \mathrm{mM} \mathrm{Mes} /$ $\mathrm{NaOH}(\mathrm{pH} 7.0)$, and assessed for biologic activity and mass spectrometry (MS) identification.

\section{Mass spectrometry}

Peptide mixtures from tryptic or $\mathrm{CNBr}$ digestions of biologically active high-performance liquid chromatography (HPLC) fractions were desalted and concentrated using C18 Zip Tips (Millipore) and analyzed by matrixassisted laser desorption/ionization time of flight mass spectrometry (MALDI-ToF MS) (Voyager DE-sSTR; Applied Biosystems, Foster City, CA) using a 337-nm wavelength laser for desorption and the reflectron mode of analysis. Mass spectra of digested peptides were searched against the FASTA database ${ }^{21}$ using the PROWL ProFound search engine ${ }^{22}$ (available at www.prowl.rockefeller.edu) using an unbiased all taxa search. For amino acid sequencing, the peptide mixtures were also analyzed by MS/MS (tandem mass spectrometry) using a Q-q-Tof hybrid system (Q-Star XL; Applied Biosystems) equipped with a nanospray ion source. In particular, the doubly charged ion of the phosphopeptide (65-86) at $\mathrm{m} / \mathrm{z} 1225.5287$ was selected to sequence and identify the Ser-77 phosphorylation site.

\section{Three-dimensional gel and migration assays}

MAE-cell aggregates were embedded in fibrin gel. ${ }^{17}$ Then, culture medium containing the chromatographic fraction to be tested or murine rDrm (R\&D Systems, Minneapolis, MN) was added on the top of the gel in the presence of $10 \mu \mathrm{g} / \mathrm{mL}$ aprotinin to prevent the dissolution of the substrate. Formation of radially growing cell sprouts was observed during the next 24 to 72 hours. Sprouts were photographed at $\times 40$ magnification (Olympus [Melville, NY] IX51 inverted microscope equipped with a $4 \times 10.10$ numerical aperture [NA] objective and a Camedia C-4040 digital camera) and quantified by computerized analysis of the digitalized images. Threedimensional gels of reconstituted rat tail tendon type I collagen fibrils (Boehringer Mannheim, Monza, Italy) were prepared as described. ${ }^{17}$ Then, BAE cells were seeded on the top of the collagen gel $\left(80000\right.$ cells $\left./ \mathrm{cm}^{2}\right)$ and allowed to reach confluence. Cell cultures were then treated with fresh medium containing rDrm plus 10\% FCS. After 24 hours, cells were photographed at $\times 100$ magnification (Olympus IX51 inverted microscope equipped with a CAch $10 \times / 0.25 \mathrm{NA}$ PhP objective), and endothelial cells invading the gel, in a plane of focus beneath the cell monolayer surface, were quantified by computerized analysis of the digitalized images. To study migration, 50000 MAE cells resuspended in DMEM plus $0.1 \%$ heat-inactivated FCS were seeded in the upper compartment of a Boyden chamber containing a gelatin-coated polycarbonate membrane filter (Nucleopore, 8 - $\mu \mathrm{m}$ pores; Whatman, Mainstone, United Kingdom). rDrm and/or BMP4 (R\&D Systems) were dissolved in the same medium and placed in the lower chamber. Then, cells were allowed to migrate for 4 hours at $37^{\circ} \mathrm{C}$. Migrated cells at the bottom surface of the filter were stained (Diff-Quick; DADE Behring, Marburg, Germany) and counted at $\times 250$ magnification (5 fields/sample in triplicate) using a Dialux $20 \mathrm{~EB}$ microscope (Leitz, Wetzlar, Germany) equipped with an NP1 $25 \times / 0.50$ NA objective.

\section{I-Drm binding and cross-linking to endothelial cells}

rDrm $(5 \mu \mathrm{g})$ was dissolved in $200 \mu \mathrm{L}$ PBS, transferred into iodogen-coated tubes (Pierce, Rockford, IL), and incubated for 5 minutes at $4^{\circ} \mathrm{C}$ with 0.2 $\mathrm{mCi}$ [7.4 MBq] ${ }^{125} \mathrm{I}$ (Amersham). The reaction products were separated on a size-exclusion Sephadex-G10 column. For ${ }^{125} \mathrm{I}-\mathrm{rDrm}$ binding experiments, SIE, MAE, and HUVE cells were plated in 24-well dishes at 70000 cells $/ \mathrm{cm}^{2}$. After 24 hours, cells were washed 3 times with ice-cold PBS and incubated for 2 hours at $4{ }^{\circ} \mathrm{C}$ in binding medium (serum-free medium containing $0.15 \%$ gelatin, $20 \mathrm{mM}$ HEPES, $\mathrm{pH}$ 7.5) with increasing concentrations of ${ }^{125} \mathrm{I}$-Drm in the absence or in the presence of 100 -fold excess of unlabeled ligand. After a PBS wash, cells were washed twice with $2.0 \mathrm{M} \mathrm{NaCl}$ in $20 \mathrm{mM}$ HEPES buffer ( $\mathrm{pH} 7.5$ ) to elute ${ }^{125} \mathrm{I}-\mathrm{rDrm}$ bound to low-affinity sites. Next, ${ }^{125} \mathrm{I}-\mathrm{rDrm}$ bound to high-affinity sites was eluted with $2.0 \mathrm{M} \mathrm{NaCl}$ in $20 \mathrm{mM}$ sodium acetate $\left(\mathrm{pH} \mathrm{4.0)} .^{23}\right.$ Low-affinity and high-affinity binding data were analyzed by Scatchard plot using Prism4 software (GraphPad Software, San Diego, CA).

For cross-linking experiment, confluent SIE and HUVE cells were incubated for 10 minutes at room temperature in binding medium with 5.0 $\mathrm{nM}{ }^{125} \mathrm{I}-\mathrm{rDrm}$ in the absence or presence of a 100 -fold molar excess of unlabeled rDrm or BMP4. Then, $1.0 \mathrm{mM}$ bis[sulphosuccinamide]suberate (Pierce) was added. The cross-linking reaction was allowed to proceed at $4^{\circ} \mathrm{C}$ for 2 hours. After a $2.0 \mathrm{M} \mathrm{NaCl}$ wash in $20 \mathrm{mM}$ HEPES buffer $(\mathrm{pH}$ 7.5 ), cells were lysed and proteins were separated by $6 \%$ sodium dodecyl sulfate-polyacrylamide gel electrophoresis (SDS-PAGE) under reducing conditions. Complexes were visualized by autoradiography of the gel.

In a second set of experiments, $10^{7}$ confluent SIE cells were incubated under the same experimental conditions with $5.0 \mathrm{nM}$ unlabeled rDrm. After 
cross-linking, cells were lysed in lysis buffer [50 mM Tris-HCl buffer $(\mathrm{pH}$ 7.4) containing $150 \mathrm{mM} \mathrm{NaCl}, 1 \%$ Triton $\mathrm{X}-100$, and protease and phosphatase inhibitors ( $50 \mu \mathrm{g} / \mathrm{mL}$ pepstatin, $50 \mu \mathrm{g} / \mathrm{mL}$ leupeptin, $10 \mu \mathrm{g} / \mathrm{mL}$ aprotinin, $1.0 \mathrm{mM} \mathrm{Na}_{3} \mathrm{VO}_{4}$; all from Sigma, St Louis, MO)], and the whole sample $(1.0 \mathrm{mg}$ protein) was incubated with anti-Drm antibody (R\&D Systems). Immunocomplexes were precipitated by overnight incubation at $4^{\circ} \mathrm{C}$ with Protein G-Sepharose beads and analyzed by Western blotting under reducing conditions using the same anti-Drm antibody. The lysate of cells in which rDrm incubation was omitted was used as a negative control.

\section{Intracellular signaling}

Confluent SIE cells were made quiescent by a 20 -hour starvation in $2 \%$ FCS. After stimulation with $\mathrm{rDrm}(50 \mathrm{ng} / \mathrm{mL})$, cells were lysed, and $20-\mu \mathrm{g}$ aliquots were analyzed by $6 \%$ or $10 \%$ SDS-PAGE followed by Western blotting with antibodies against pFAK, pPaxillin, pERK $_{1 / 2}$, or Jak2 (Santa Cruz Biotechnology, Santa Cruz, CA). For 2D-PAGE, samples were solubilized in $8.0 \mathrm{M}$ urea, $50 \mathrm{mM}$ DTT, $4 \%$ CHAPS, $0.2 \%$ carrier ampholytes (pH 3-10), 0.0002\% bromophenol blue. Proteins were separated on pH 3-10 NL IPG strips (Bio-Rad, Munich, Germany) according to the manufacturer's instructions. The second dimension was performed on $10 \%$ SDS-PAGE, and proteins were probed with anti-pTyr antibody (Santa Cruz Biotechnology) in a Western blot. The same antibody was used to decorate rDrm-stimulated, paraformaldehyde-fixed SIE cells.

\section{Drm/gremlin transfection in COS cells}

COS cells were transfected with the pMEXneo expression vector harboring the rat Drm cDNA (pMEX-DRM) or with the empty vector (both provided by D. G. Blair, National Cancer Institute [NCI]-Frederick, MD) to generate stable transfectants (mock-COS cells and Drm-COS cells, respectively) as described. ${ }^{24}$

\section{Chicken embryo chorioallantoic membrane (CAM) assay}

Gelatin sponges containing vehicle, 50 or $100 \mathrm{ng}$ of rDrm or BMP4 or both ( $\mathrm{n}=10-20 \mathrm{eggs} / \mathrm{group}$ ), were placed on the CAM of fertilized chicken eggs at day 8 of incubation..$^{25}$ In a parallel experiment, mock-COS cells and Drm-COS cells were implanted via a gelatin sponge (18 000 cells/sponge) on the top of the CAM at day 8 ( $\mathrm{n}=20 \mathrm{eggs} /$ group $)$. At day 12 , blood vessels entering the sponge within the focal plane of the CAM were counted at $\times 50$ magnification using a STEMI SR stereomicroscope equipped with an objective f equal to $100 \mathrm{~mm}$ with adapter ring 475070 (Carl Zeiss, Jena, Germany). When indicated, CAMs were processed for light microscopy, and microvessel density was evaluated by a planimetric method. ${ }^{25}$

\section{Drm/gremlin expression in tumor xenografts}

Drm/gremlin transcripts were evaluated by reverse-transcription-polymerase chain reaction (RT-PCR) analysis in human endometrial adenocarcinoma HEC-1-B-derived xenografts ${ }^{26}$ (provided by R. Giavazzi, Mario Negri Institute, Bergamo, Italy) using the following specie-specific primers: murine Drm/gremlin [(+)CTCAGCACAATGACTCCGAGC; (-)ATCCAAGTCGATGGATATGCAA], human Drm/gremlin [(+)GTATGAGCCGCACAGCCTACA; (-)CTCGCTTCAGGTATTTGCGCT]. After PCR reaction, $5-\mu \mathrm{L}$ aliquots were separated on a $1.5 \%$ agarose gel and visualized by ethidium bromide staining. Also, paraffin-embedded tumor samples were stained with anti-Drm/gremlin antibody as detailed in the next paragraph.

\section{Human lung tumor collection and immunohistochemistry}

Ten samples of formalin-fixed and paraffin-embedded human lung cancers (5 adenocarcinomas and 5 squamous-cell carcinomas) were obtained from L. de Leval (Department of Pathology, Liège University Hospital, Belgium). Paraffin sections $(5 \mu \mathrm{m})$ were stained with a goat polyclonal anti-Drm/gremlin antibody (R\&D Systems) using the immunoperoxidase $\mathrm{ABC}$ Vectastain Kit (Vector Laboratories, Burlingame, CA). Antigen retrieval was performed by heating slides in a water-bath at $95^{\circ} \mathrm{C}$ for 40 minutes in $10 \mathrm{mM}$ citrate buffer ( $\mathrm{pH} \mathrm{6.0).} \mathrm{After} \mathrm{blocking} \mathrm{the} \mathrm{endoge-}$ nous peroxidase activity with $0.3 \% \mathrm{H}_{2} \mathrm{O}_{2}$ in methanol for 30 minutes, slides were sequentially incubated with normal horse serum (1:20) for 30 minutes and with the anti-gremlin antibody $(1: 30)$ overnight at $4{ }^{\circ} \mathrm{C}$. Slides were then incubated with biotinylated antigoat antibody (Vector Laboratories) followed by the avidin-biotin-peroxidase complex. Hematoxylincounterstained sections were reviewed by 2 independent observers, and immunostaining intensity in cancer cells and in endothelial cells was scored as follows: - indicates negative; + , weak staining; ++ , strong staining. Analysis of tissue sections was performed using a Zeiss Axiovert $200 \mathrm{M}$ microscope equipped with an Achroplan $40 \times 10.65 \mathrm{NA} \mathrm{Ph} 2$ or an Achroplan $20 \times / 0.45$ NA objective.

\section{Data representation}

Data are expressed as mean \pm SD. Statistical analyses were done using the Student $t$ test. The significance level was set at $P$ values less than .01 .

\section{Results}

\section{Drm/gremlin purification}

Preliminary observations had shown that FGF2-T-MAE cells release an uncharacterized heparin-binding, heat-stable, proteinaceous factor responsible for the capacity of their CM to induce endothelial-cell sprouting in a fibrin gel. ${ }^{19}$ On this basis, we thought to purify this protein sequentially using ammonium sulfate precipitation, cation exchange chromatography, heparin-Sepharose affinity chromatography, and reversed-phase HPLC (Figure 1). The bioactivity of eluted fractions was monitored by stimulation of MAE-cell sprouting in 3-dimensional fibrin gel (Figure 1A). Most of the endothelial-cell sprouting-inducing activity present in the serum-free CM of FGF2-T-MAE cells precipitates in 70\% ammonium sulfate and binds to an SP-Sepharose cation exchange column from where it is recovered in the 0.8 to $0.9 \mathrm{M} \mathrm{NaCl}$ eluate (Figure 1B). Subsequent fractionation by heparin-Sepharose chromatography reveals a major peak of activity eluting at $1.0 \mathrm{M} \mathrm{NaCl}$ (Figure 1C). Further purification by reversed-phase HPLC yields 2 biologically active peaks (retention time of 24.6 and 26.7 minutes; Figure 1D). Peptide mass fingerprinting analysis of both peaks by MALDIToF mass spectrometry (MS) (Figure 1E) identifies the purified endothelial-cell motogen as the murine Drm gene product. The identification was confirmed by nano-ESI-MS/MS sequencing of the peptides generated by tryptic and $\mathrm{CNBr}$ digestion of the purified protein and by Western blotting of the bioactive HPLC fractions (Figure 1D, inset).

Nano-ESI-MS/MS analysis also shows that purified Drm undergoes posttranslational modifications, including maturation, glycosylation, and phosphorylation, in keeping with previous observations in Drm-transfected COS cells. ${ }^{24}$ Indeed, purified Drm lacks the leader sequence for secretion (amino acid sequence starting from Lys-25) and carries one N-glycosylation at Asn-42 and one phosphorylation at Ser-77 (Figure 1F). These posttranslational modifications explain the presence of more Drm forms after HPLC and Western blot analysis of the bioactive fractions (Figure 1D).

\section{Drm/gremlin as an endothelial-cell motogen}

To confirm the ability of Drm/gremlin to induce a motogenic response in endothelial cells, increasing concentrations of recombinant murine Drm (rDrm) were tested for the capacity to stimulate the sprouting of MAE-cell aggregates in fibrin gel, the chemotactic response of MAE cells in a Boyden chamber assay, and the 
Figure 1. Drm/gremlin purification from the CM of FGF2-TMAE cells. (A) FGF2-T-MAE-cell CM (bottom), but not MAEcell CM (top), stimulates sprouting of MAE-cell aggregates in fibrin gel. This assay was used to follow the purification of FGF2-T-MAE-cell CM by cation exchange (B) and heparinSepharose (C) chromatography (horizontal bar, pooled bioactive fractions; diagonal dashed lines, $\mathrm{NaCl}$ gradient), and by reverse-phase HPLC (D). Bioactive HPLC peaks $\left({ }^{*},{ }^{* *}\right)$ were probed with anti-Drm/gremlin antibodies in a Western blot (D, inset) and identified by MALDI-ToF peptide mass fingerprinting (peak * in panel E; peak ${ }^{*}$, not shown). (F) Amino acid sequence of Drm/gremlin. Tryptic (underlined) or $\mathrm{CNBr}$ (italic) digestion peptides were sequenced by nano-ESI-MS/MS. $\mathrm{N}$-glycosylation $(\mathrm{N})$ and Ser-phosphorylation (S) sites are highlighted.

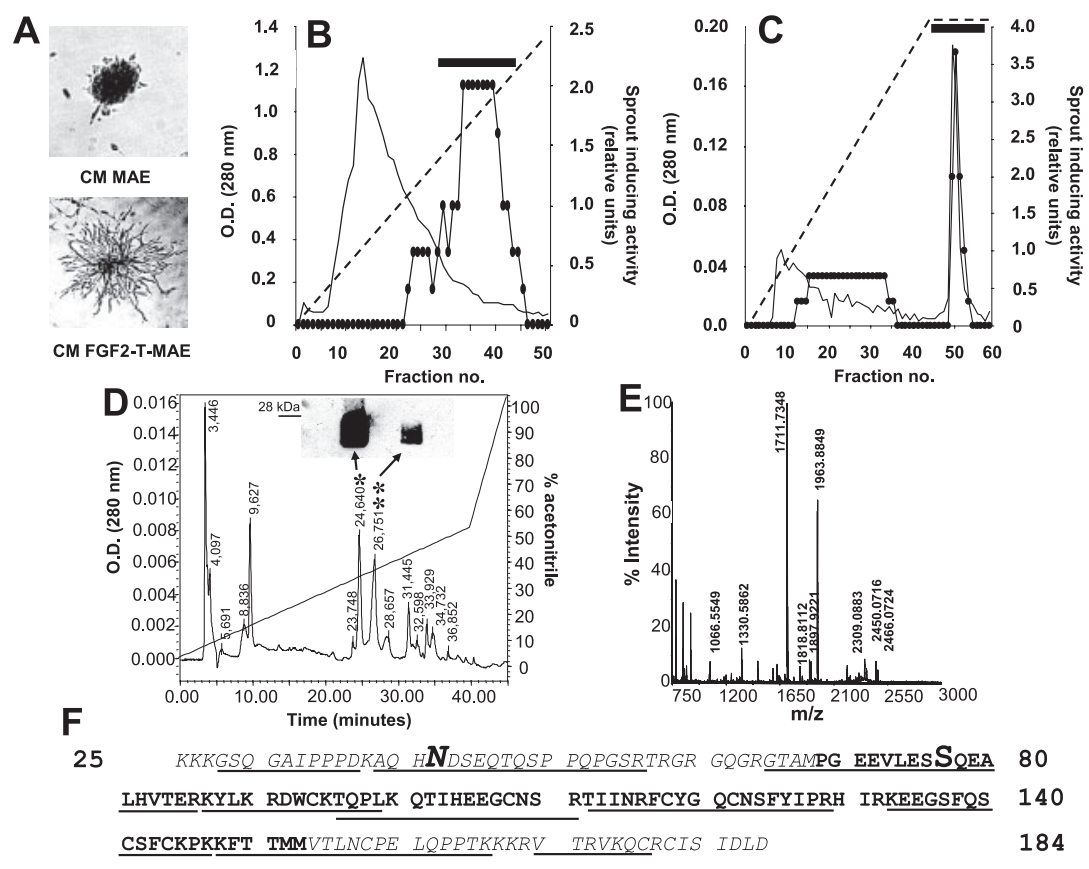

invasion of type I collagen gel by BAE cells (Figure 2A). In all the assays, rDrm stimulates endothelial-cell motility with a maximal response at approximately $50 \mathrm{ng} / \mathrm{mL}$. In keeping with its motogenic activity, rDrm stimulates tyrosine phosphorylation of focal adhesion contacts (Figure 2A, inset) and phosphorylation of focal adhesion kinase (FAK) (Figure 3B) in murine dermal microvascular endothelial (SIE) cells. Taken together, the data identify Drm/gremlin as a motility factor for endothelial cells of different origin.

\section{Drm/gremlin binding to endothelial cells}

The capacity of rDrm to stimulate endothelial-cell motility and to induce rapid intracellular phosphorylation events points to the previously unrecognized possibility that Drm/gremlin may interact
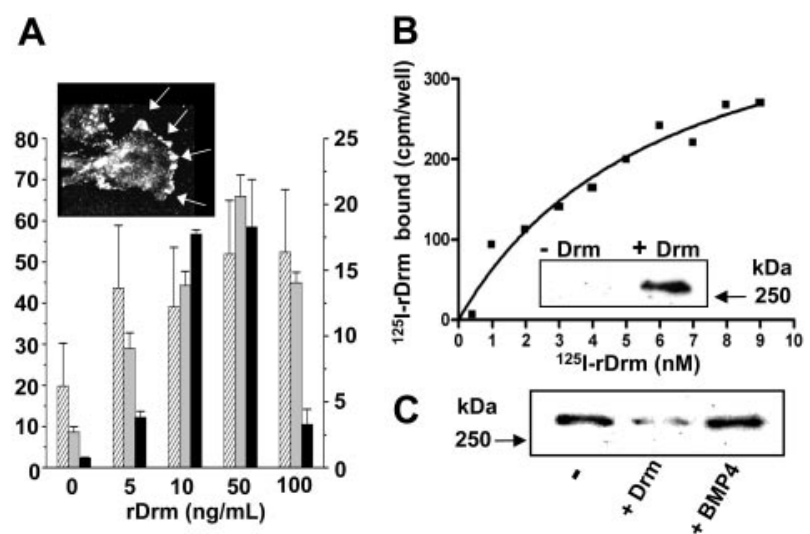

Figure 2. rDrm interaction with endothelial cells. (A) rDrm induces MAE-cell sprouting in fibrin gel (四), MAE-cell migration in a Boyden chamber (圆), and BAE-cell invasion in type I collagen gel ( $\square$ ) (data expressed as mean \pm SD); inset, tyrosine phosphorylation of focal adhesion contacts (arrows) in SIE cells stimulated for 10 minutes with $100 \mathrm{ng} / \mathrm{mL} \mathrm{rDrm}$. Cell analysis was performed using a Zeiss Axiovert $200 \mathrm{M}$ microscope equipped with a Plan-Apochromat $63 \times / 1.4$ NA oil objective. (B) Binding of ${ }^{125} \mathrm{I}-\mathrm{rDrm}$ to SIE cells; inset, cross-linking of unlabeled rDrm to SIE cells followed by immunoprecipitation and Western blotting with anti-Drm antibody. (C) Cross-linking of ${ }^{125} \mathrm{I}-\mathrm{rDrm}$ to SIE cells with or without a 100 -fold molar excess of unlabeled rDrm or BMP4. ${ }^{125} \mathrm{I}-\mathrm{rDrm}$ complexes were visualized by autoradiography of the SDS-PAGE gel. directly with endothelial cells. Indeed, Scatchard plot analysis of the binding data of ${ }^{125} \mathrm{I}-\mathrm{rDrm}$ to different endothelial cell types (including SIE, MAE, and HUVE cells) revealed one class of high-affinity binding sites with a $\mathrm{K}_{\mathrm{d}}$ of $5.1 \pm 0.3 \mathrm{nM}$ and a $\mathrm{B}_{\max }$ of $7000 \pm 1700$ binding sites/cell (Figure 2B). As observed for other heparin-binding angiogenic factors, ${ }^{27}{ }^{125} \mathrm{I}$-rDrm interacts also with one class of low-affinity binding sites on the surface of the different endothelial cell types (from 80000 to 250000 binding sites/cell) with a $\mathrm{K}_{\mathrm{d}}$ ranging between $30 \mathrm{nM}$ and $300 \mathrm{nM}$. The capacity of free heparin to fully prevent this binding (data not shown) is consistent with the hypothesis that Drm/gremlin may engage a low-affinity interaction with cell-surface heparan-sulfate proteoglycans. ${ }^{28}$

Cross-linking of $\mathrm{rDrm}$ to the cell surface followed by immunoprecipitation with anti-Drm antibody shows that rDrm binds to a cell-membrane protein, originating a Drm-immunoreactive protein complex with a molecular mass of approximately $250 \mathrm{kDa}$ (Figure 2B, inset). Remarkably, ${ }^{125} \mathrm{I}-\mathrm{rDrm} /$ receptor complex formation is prevented by a molar excess of unlabeled rDrm but not of the Drm/gremlin ligand BMP4

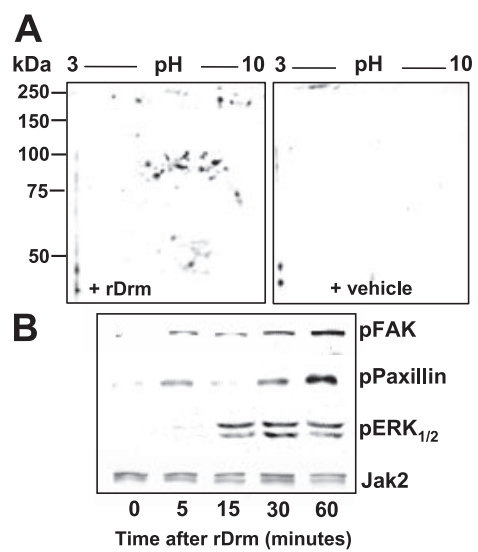

Figure 3. rDrm-induced intracellular signaling. (A) Two-dimensional electrophoresis of control and rDrm-treated SIE-cell extracts decorated with anti-phospho-Tyr antibody. (B) FAK, paxillin, and $\mathrm{ERK}_{1 / 2}$ phosphorylation in rDrm-treated SIE cells. Anti-Jak2 antibody was used as loading control. 
(Figure 2C), indicating that BMP interaction does not hamper the receptor-binding capacity of $\mathrm{rDrm}$.

In keeping with the presence of a cell-surface receptor, rDrm causes the rapid appearance of a composite tyrosine phosphorylation pattern in endothelial SIE cells (Figure 3A). As anticipated for a cell-motility factor, paxillin, FAK, and mitogenactivated protein kinase $\mathrm{ERK}_{1 / 2}$ are targets of rDrm-activated signaling (Figure 3B). Taken together, the data demonstrate for the first time the capacity of Drm/gremlin to exert a direct, productive interaction with endothelial cells by engaging cellsurface receptor(s) whose identification will require further investigation.

\section{Angiogenic activity of Drm/gremlin}

To assess the effect of Drm/gremlin on blood vessel formation in vivo, rDrm (100 ng/egg) was delivered on the top of the chick embryo CAM at day 8 of development via a gelatin sponge implant ${ }^{25} \mathrm{~A}$ potent angiogenic response was observed around the rDrm implants when compared with vehicle-treated embryos (Figure 4A-B), the number of macroscopic blood vessels converging toward the gelatin sponge being significantly higher $(P<.01)$ in rDrm than in vehicle implants (Table 1). Histologic morphometric analysis of microvessel density within the gelatin implants confirmed the proangiogenic activity of rDrm (Figure 4C-D; Table 1). To substantiate these observations, we transfected COS cells with an expression vector harboring the rat Drm cDNA. Transfectants produce and release significant amounts of the recombinant protein (Figure S1, available on the Blood website; see the Supplemental Figure link at the top of the online article). Accordingly, a potent angiogenic response was observed in chick embryo CAMs implanted with Drm-transfectants when compared with mock-transfected cells (Figure S1; Table 1).

\section{Effect of BMP4 on the angiogenic activity of Drm/gremlin}

Our data indicate that rDrm binds to endothelial cells in a BMP-independent manner (see Figure 2C). However, previous

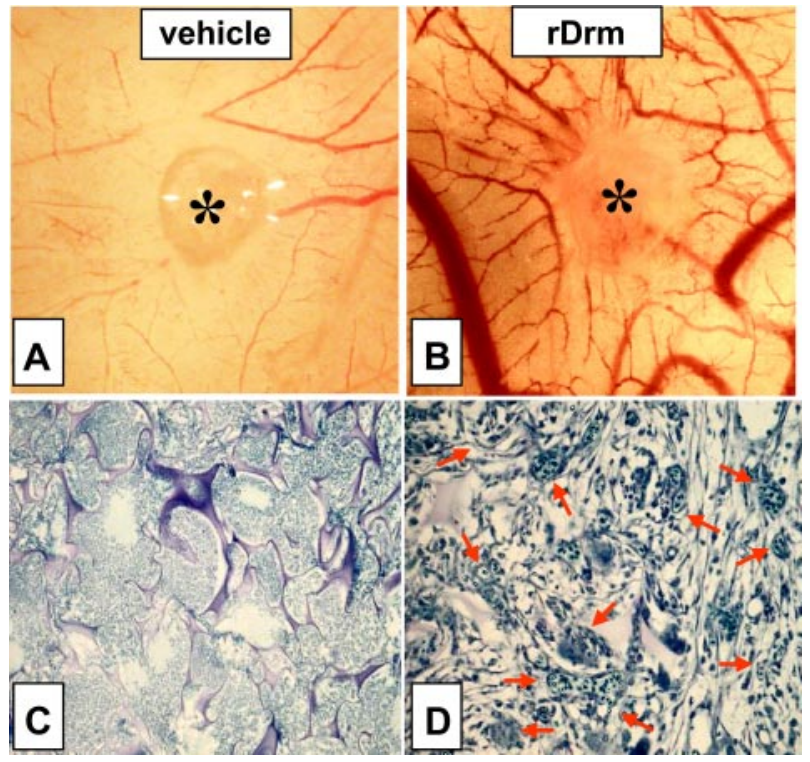

Figure 4. Proangiogenic activity of rDrm. Chick embryo CAM implants (asterisk indicates gelatin sponge) were loaded with vehicle (A) or $100 \mathrm{ng}$ rDrm (B). Note the numerous blood vessels converging versus the rDrm implant. (C-D) Toluidine blue staining of histologic sections of CAM treated with vehicle (C) or $100 \mathrm{ng}$ rDrm (D). Numerous capillaries filled with nucleated avian erythrocytes are present among the trabeculae of the rDrm-treated sponge (arrows in panel D).
Table 1. Chick embryo CAM assay: macroscopic and microscopic assessment of Drm-induced neovascularization

\begin{tabular}{lcccc}
\hline \multicolumn{1}{c}{ Treatment } & $\mathbf{n}$ & $\begin{array}{c}\text { Blood vessels, } \\
\text { mean } \pm \text { SD }\end{array}$ & $\begin{array}{c}\text { Intersection points, } \boldsymbol{\dagger} \\
\text { mean } \pm \text { SD }\end{array}$ & $\begin{array}{c}\text { Microvessel } \\
\text { density, } \boldsymbol{\dagger} \%\end{array}$ \\
\hline Vehicle & 10 & $6 \pm 3$ & 0 & 0.0 \\
rDrm & 20 & $27 \pm 4 \ddagger$ & $23 \pm 3$ & 16.0 \\
Mock-COS cells & 20 & $10 \pm 3$ & $5 \pm 2$ & 3.5 \\
Drm-COS cells & 20 & $28 \pm 3 \ddagger$ & $24 \pm 2$ & 16.6 \\
\hline
\end{tabular}

Gelatin sponges containing vehicle, 100 ng rDrm, mock-transfected or Drmtransfected COS cells (18 000 cells per sponge) were implanted on top of chick embryo CAMs at day 8.

*The angiogenic response was assessed macroscopically at day 12 by counting the number of blood vessels entering the sponge.

†The angiogenic response was assessed histologically by a planimetric method of "point counting" as described. ${ }^{24}$

$\ddagger P<.001$ versus vehicle and Mock-COS cells.

observations had shown that BMPs are endowed with angiogenic activity. ${ }^{29,30}$ Accordingly, the Drm/gremlin ligand BMP4 is a chemoattractant for MAE cells and causes neovascularization in the chick CAM (Figure 5A-B). On this basis, we investigated the effect of BMP4 on the angiogenic activity of rDrm in vitro and in vivo. As shown in Figure 5A, stimulation of MAE cells with a suboptimal concentration of $\mathrm{rDrm}(5.0 \mathrm{ng} / \mathrm{mL})$ in the presence of increasing concentrations of BMP4 resulted in an increased chemotactic response. Similarly, the simultaneous delivery of rDrm and BMP4 on the chick CAM triggered a more potent angiogenic response when compared with that induced by rDrm alone (Figure 5B).Taken together, these observations support the hypothesis that Drm/gremlin exerts its angiogenic activity on endothelium via a BMP-independent mechanism of action.

\section{Drm/gremlin expression in tumor vasculature}

Recent observations have shown that Drm/gremlin is up-regulated in human cancers. ${ }^{31}$ To gain insights about a possible role of Drm/gremlin in tumor vascularization, we performed immunohistochemical analysis of experimental and human tumors. Highly vascularized tumor xenografts generated by subcutaneous injection in nude mice of human adenocarcinoma HEC-1-B-derived cells ${ }^{26}$ showed a strong Drm immunoractivity in the tumor stroma, whereas tumor parenchyma was negative (Figure 6A-B). RT-PCR analysis of total RNA from these lesions using specie-specific primers confirmed the murine stromal origin of Drm/gremlin and the lack of human Drm/gremlin transcripts (Figure 6).

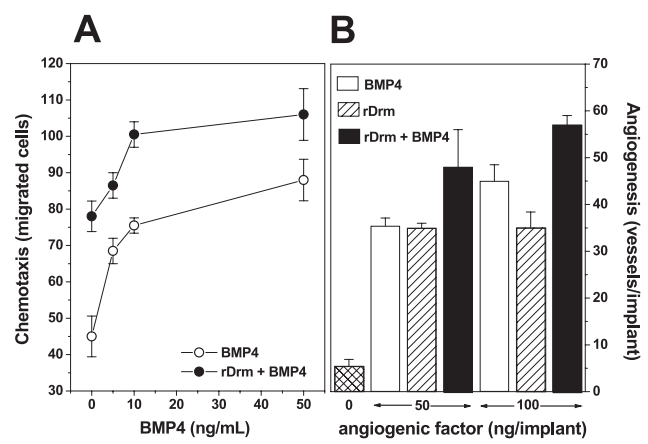

Figure 5. Effect of BMP4 on the angiogenic activity of Drm/gremlin. (A) The chemotactic activity of a suboptimal concentration of $\mathrm{rDrm}(5.0 \mathrm{ng} / \mathrm{mL})$ was tested on MAE cells in a Boyden chamber assay in the presence of increasing concentrations of BMP4 (O); in parallel, the chemotactic activity of BMP4 alone was tested on the same cells $(O)$. (B) Chick embryo CAM implants were loaded with vehicle, BMP4, rDrm, or rDrm plus BMP4 (both molecules were tested alone or in combination at 50 or $100 \mathrm{ng} / \mathrm{implant}$ ). After 4 days, blood vessels converging versus the implant were counted. Data are expressed as mean \pm SD. 

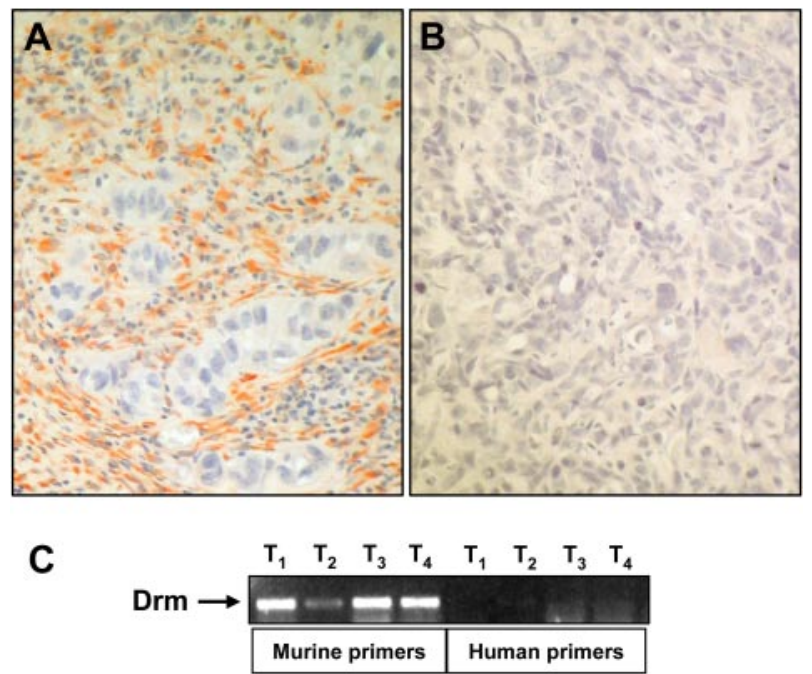

Figure 6. Drm/gremlin expression in tumor xenografts. (A) Drm/gremlin immunostaining of tumor xenografts originated in nude mice by subcutaneous injection of human endometrial adenocarcinoma HEC-1-B-derived cells. Note the Drm/gremlin immunoreactivity in tumor stroma, absent in tumor parenchyma. (B) Negative control in which the primary antibody was omitted. Original magnification, $\times 200$. (C) RT-PCR analysis of 4 tumor xenografts $(\mathrm{T})$ using murine and human specific Drm/gremlin primers.

The cancer profiling DNA microarray database Oncomine 2.0 contains publicly available gene expression sets generated from approximately 60 tumor panels covering 20 different human malignancies. ${ }^{32}$ Database mining reveals that Drm is significantly overexpressed in lung adenocarcinoma and squamous-cell carcinoma specimens when compared with normal lung $(P<.001)$. Accordingly, immunostaining of 10 lung cancer samples (Table 2) demonstrated that endothelial cells of vessels infiltrating or adjacent to tumor cells strongly express Drm/gremlin (Figure 7A-C). Cancer cells lacked Drm/gremlin expression in 7 cases and showed a weak cytoplasmic immunostaining in the remaining 3 cases (Table 2). In all cases, endothelium of the nonneoplastic lung tissue adjacent to tumor lesions showed no or very weak immunoreactivity (Figure 7D).

\section{Discussion}

Our results show for the first time the capacity of Drm/gremlin to induce a motile and invasive phenotype in endothelial cells in vitro and a potent angiogenic response in vivo. This reflects the previously unrecognized ability of Drm/gremlin to interact with

Table 2. Immunohistochemical analysis of Drm/gremlin expression in human lung tumors

No. of tumors with the indicated Drm/gremlin immunostaining intensity

\begin{tabular}{|c|c|c|c|c|c|c|}
\hline \multirow[b]{3}{*}{ Lung tumors } & \multicolumn{6}{|c|}{ immunostaining intensity } \\
\hline & \multicolumn{3}{|c|}{$\begin{array}{c}\text { Cancer-cell } \\
\text { immunostaining }\end{array}$} & \multicolumn{3}{|c|}{$\begin{array}{l}\text { Endothelial-cell } \\
\text { immunostaining }\end{array}$} \\
\hline & - & + & ++ & - & + & ++ \\
\hline Adenocarcinomas & 4 & 1 & 0 & 0 & 0 & 5 \\
\hline Squamous-cell carcinomas & 3 & 2 & 0 & 0 & 1 & 4 \\
\hline
\end{tabular}

Paraffin-embedded human lung cancer specimens (for each type listed in the table, $n=5$ ) were analyzed for Drm/gremlin expression by immunostaining. Cancer cells and endothelial cells of each specimen were scored for Drm/gremlin immunoreactivity on an arbitrary scale: - indicates negative; + , weak staining; ++ , strong staining.
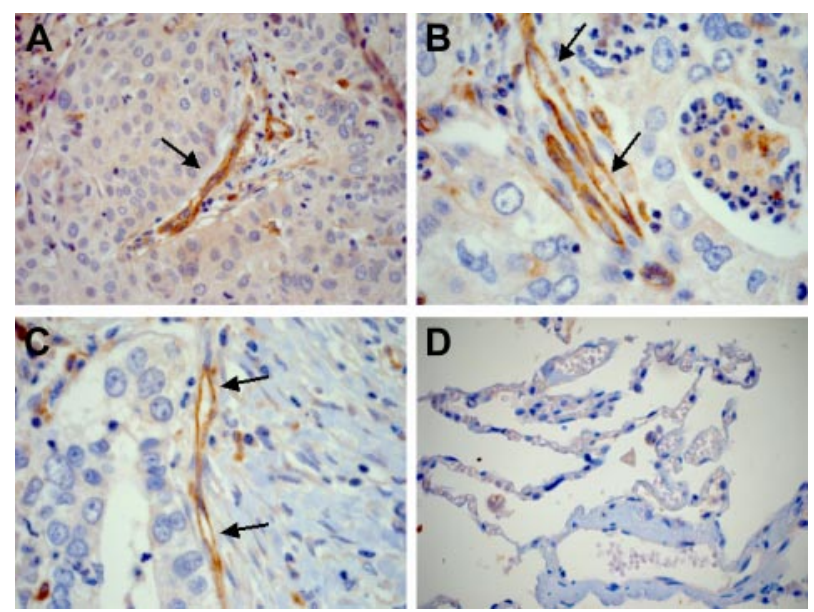

Figure 7. Drm/gremlin immunohistochemistry in human lung cancers. Endothelial cells of tumor vessels (arrows) show a strong Drm/gremlin immunoreactivity in human lung squamous-cell carcinoma (A) and adenocarcinoma (B-C). Squamous tumor cells are weakly immunoreactive $(A)$. No or very weak immunoreactivity is detected in nonneoplastic lung tissue adjacent to the tumor (D). Original magnification, $\times 200(A, D) ; \times 400(B, C)$.

high-affinity binding sites on endothelial cells in a BMPindependent manner and to trigger a complex intracellular signaling cascade.

Drm/gremlin exerts a BMP antagonist activity by binding BMP2, BMP4, and BMP7. ${ }^{3}$ Previous observations had shown that BMP2 and BMP4 are endowed with angiogenic activity. ${ }^{29,30}$ Here, we have confirmed these observations by showing that BMP4 exerts a significant angiogenic response in the chick embryo CAM. However, BMP4 does not hamper Drm/gremlin interaction with endothelial-cell receptors, and the simultaneous exposure of endothelium to both rDrm and BMP4 results in a potentiated chemotactic and angiogenic response in vitro and in vivo, respectively. Our observations support the hypothesis that Drm/gremlin exerts its angiogenic activity on endothelium via a BMP-independent mechanism of action. Further experiments are required to fully dissect the complex interplay among Drm/gremlin, BMPs, and their cognate receptors.

Drm/gremlin is overexpressed in human cancers. ${ }^{31}$ We have found that human tumor xenografts in nude mice express Drm. Drm transcripts are synthesized by cells of the host within the tumor, as shown by RT-PCR analysis using specific murine primers, and Drm/gremlin protein accumulates in the stroma of the tumor. Also, we have observed a strong Drm/gremlin immunoreactivity in endothelial cells of human lung tumor samples, absent in adjacent nonneoplastic lung tissue. Recombinant FGF2 up-regulates Drm expression in endothelial cells of different origin (M.P., unpublished observations, October 2006). This is in keeping with the capacity of FGF2, as well as of BMP2 and platelet-derived growth factor, to induce Drm/ gremlin production in rat osteoblasts ${ }^{33}$ and with the role of Drm/gremlin in the sonic hedgehog/FGF feedback loop in vertebrate limb bud. ${ }^{34}$ The possibility that Drm/gremlin produced by tumor cells and/or by growth factor-activated endothelium may contribute to neovascularization in experimental and human neoplasms deserves further investigation.

Secreted Drm/gremlin binds to various BMPs. ${ }^{3}$ Also, Drm/ gremlin has been shown to bind Slit proteins and to act as inhibitor of monocyte chemotaxis. ${ }^{8}$ Moreover, a possible intracellular, BMP-independent mechanism of action has been hypothesized for this protein. ${ }^{6}$ Our observations point out the 
previously unrecognized capacity of Drm/gremlin to interact with signaling receptor(s) in endothelial cells with possible implications in angiogenesis. Thus, Drm/gremlin may exert multiple functions by interacting with different partners. The ability of Drm/gremlin to act directly on target endothelial cells via a receptor-mediated mechanism of action sheds new light on its putative role in embryogenesis and human diseases, including cancer.

\section{Acknowledgments}

We thank M. L. Massardi and S. Palermo for technical assistance and M. Rusnati and P. Dell'Era (University of Brescia) and A. Scarpa (University of Verona) for helpful discussion and criticisms.

This work was supported by grants from Ministero dell' Istruzione dell'Università e della Ricerca (MIUR) (Centro di
Eccellenza, Centro per l'Innovazione Diagnostica e Terapeutica [IDET], Fondo per gli Investimenti della Ricerca di Base [FIRB] 2001, Cofin 2004), Fondazione Berlucchi, Istituto Superiore Sanità (Progetto Oncotecnologico), and Associazione Italiana per la Ricerca sul Cancro (AIRC) (M.P.).

\section{Authorship}

Contribution: H.S., S.M., F.T., and M.P. designed the research; H.S., S.M., E.M., M.B., S.N., D.C., L.O., V.C., D.W., and D.R. performed the research; H.S., S.M., F.P., A.P., F.C., and M.P. analyzed the data; and S.M. and M.P. wrote the paper.

Conflict-of-interest disclosure: The authors declare no competing financial interests.

Correspondence: Marco Presta, General Pathology, Department of Biomedical Sciences and Biotechnology, University of Brescia, Viale Europa 11, 25123 Brescia, Italy; e-mail: presta@med.unibs.it.

\section{References}

1. Pearce JJ, Penny G, Rossant J. A mouse cerberus/Dan-related gene family. Dev Biol. 1999;209: 98-110.

2. Vitt UA, Hsu SY, Hsueh AJ. Evolution and classification of cystine knot-containing hormones and related extracellular signaling molecules. Mol Endocrinol. 2001;15:681-694.

3. Balemans W, Van Hul W. Extracellular regulation of BMP signaling in vertebrates: a cocktail of modulators. Dev Biol. 2002;250:231-250.

4. Khokha MK, Hsu D, Brunet LJ, Dionne MS, Harland RM. Gremlin is the BMP antagonist required for maintenance of Shh and Fgf signals during limb patterning. Nat Genet. 2003;34:303-307.

5. Lappin DW, McMahon R, Murphy M, Brady HR. Gremlin: an example of the re-emergence of developmental programmes in diabetic nephropathy. Nephrol Dial Transplant. 2002;17(suppl 9): 65-67.

6. Chen B, Athanasiou M, Gu Q, Blair DG. Drm/ Gremlin transcriptionally activates p21(Cip1) via a novel mechanism and inhibits neoplastic transformation. Biochem Biophys Res Commun. 2002; 295:1135-1141.

7. Topol LZ, Marx M, Laugier D, et al. Identification of drm, a novel gene whose expression is suppressed in transformed cells and which can inhibit growth of normal but not transformed cells in culture. Mol Cell Biol. 1997;17:4801-4810.

8. Chen B, Blair DG, Plisov S, et al. Cutting edge: bone morphogenetic protein antagonists Drm/ Gremlin and Dan interact with Slits and act as negative regulators of monocyte chemotaxis. J Immunol. 2004;173:5914-5917.

9. Carmeliet $P$, Jain RK. Angiogenesis in cancer and other diseases. Nature. 2000;407:249-257.

10. Hanahan D, Christofori G, Naik P, Arbeit J. Transgenic mouse models of tumour angiogenesis: the angiogenic switch, its molecular controls, and prospects for preclinical therapeutic models. Eur J Cancer. 1996;32A:2386-2393.

11. Folkman J. Angiogenesis in cancer, vascular, rheumatoid and other disease. Nat Med. 1995;1: 27-31.

12. Ferrara N. VEGF and the quest for tumour angio- genesis factors. Nat Rev Cancer. 2002;2:795803.

13. Carmeliet P. VEGF as a key mediator of angiogenesis in cancer. Oncology. 2005;69(suppl 3):410.

14. Presta M, Urbinati C, Dell'era $P$, et al. Expression of basic fibroblast growth factor and its receptors in human fetal microglia cells. Int J Dev Neurosci. 1995;13:29-39.

15. Javerzat S, Auguste P, Bikfalvi A. The role of fibroblast growth factors in vascular development. Trends Mol Med. 2002;8:483-489.

16. Sola F, Gualandris A, Belleri M, et al. Endothelial cells overexpressing basic fibroblast growth factor (FGF-2) induce vascular tumors in immunodeficient mice. Angiogenesis. 1997;1:102-116.

17. Gualandris A, Rusnati M, Belleri M, et al. Basic fibroblast growth factor overexpression in endothelial cells: an autocrine mechanism for angiogenesis and angioproliferative diseases. Cell Growth Differ. 1996;7:147-160.

18. Dell'Era P, Coco L, Ronca R, Sennino B, Presta M. Gene expression profile in fibroblast growth factor 2-transformed endothelial cells. Oncogene. 2002;21:2433-2440.

19. Ribatti D, Gualandris A, Belleri M, et al. Alterations of blood vessel development by endothelial cells overexpressing fibroblast growth factor-2. J Pathol. 1999;189:590-599.

20. Dong QG, Bernasconi S, Lostaglio S, et al. A general strategy for isolation of endothelial cells from murine tissues: characterization of two endothelial cell lines from the murine lung and subcutaneous sponge implants. Arterioscler Thromb Vasc Biol. 1997; 17:1599-1604.

21. National Center for Biotechnology Information FASTA. http://prowl.rockefeller.edu. Accessed March 2005.

22. Zhang W, Chait BT. ProFound: an expert system for protein identification using mass spectrometric peptide mapping information. Anal Chem. 2000; 72:2482-2489.

23. Coltrini D, Rusnati M, Zoppetti G, et al. Different effects of mucosal, bovine lung and chemically modified heparin on selected biological properties of basic fibroblast growth factor. Biochem J. 1994;303:583-590.

24. Topol LZ, Bardot B, Zhang Q, et al. Biosynthesis, post-translation modification, and functional characterization of Drm/Gremlin. J Biol Chem. 2000; 275:8785-8793.

25. Ribatti D, Gualandris A, Bastaki M, et al. New model for the study of angiogenesis and antiangiogenesis in the chick embryo chorioallantoic membrane: the gelatin sponge/chorioallantoic membrane assay. J Vasc Res. 1997;34:455-463.

26. Giavazzi R, Sennino B, Coltrini D, et al. Distinct role of fibroblast growth factor-2 and vascular endothelial growth factor on tumor growth and angiogenesis. Am J Pathol. 2003;162:1913-1926.

27. Iozzo RV, San Antonio JD. Heparan sulfate proteoglycans: heavy hitters in the angiogenesis arena. J Clin Invest. 2001;108:349-355.

28. Presta M, Leali D, Stabile $\mathrm{H}$, et al. Heparin derivatives as angiogenesis inhibitors. Curr Pharm Des. 2003;9:553-566.

29. Nimmagadda S, Geetha Loganathan P, Huang R, Scaal M, Schmidt C, Christ B. BMP4 and noggin control embryonic blood vessel formation by antagonistic regulation of VEGFR-2 (Quek1) expression. Dev Biol. 2005;280:100-110.

30. Langenfeld EM, Langenfeld J. Bone morphogenetic protein-2 stimulates angiogenesis in developing tumors. Mol Cancer Res. 2004;2:141-149.

31. Namkoong H, Shin SM, Kim HK, et al. The bone morphogenetic protein antagonist gremlin 1 is overexpressed in human cancers and interacts with YWHAH protein. BMC Cancer. 2006;6:74.

32. Rhodes DR, Yu J, Shanker K, et al. ONCOMINE: a cancer microarray database and integrated data-mining platform. Neoplasia. 2004;6:1-6.

33. Pereira RC, Economides AN, Canalis E. Bone morphogenetic proteins induce gremlin, a protein that limits their activity in osteoblasts. Endocrinology. 2000;141:4558-4563.

34. Zuniga A, Haramis AP, McMahon AP, Zeller R Signal relay by BMP antagonism controls the SHH/FGF4 feedback loop in vertebrate limb buds. Nature. 1999;401:598-602. 